\title{
A novel telomerase substrate precursor rapidly induces telomere dysfunction in telomerase positive cancer cells but not telomerase silent normal cells
}

\author{
Ilgen Mender ${ }^{1,2}$, Sergei Gryaznov ${ }^{3}$, and Jerry W. Shay ${ }^{1,4}$ \\ ${ }^{1}$ University of Texas Southwestern Medical Center, Department of Cell Biology, Dallas, TX, USA \\ ${ }^{2}$ Hacettepe University, Faculty of Medicine, Department of Biochemistry, Ankara, Turkey \\ ${ }^{3}$ AuraSense Therapeutics, Skokie, IL, USA \\ ${ }^{4}$ Center of Excellence in Genomic Medicine Research, King Abdulaziz University, Jeddah, Kingdom of Saudi Arabia \\ Correspondence to: Jerry W. Shay, email: Jerry.Shay@UTSouthwestern.edu \\ Keywords: cancer, telomere shortening, telomere induced foci, 6-thioguanine, 6-thio-2' deoxyguanosine \\ Received: July 08, 2015 \\ Accepted: August 17, 2015 \\ Published: August 22, 2015
}

This is an open-access article distributed under the terms of the Creative Commons Attribution License, which permits unrestricted use, distribution, and reproduction in any medium, provided the original author and source are credited.

\section{ABSTRACT}

\begin{abstract}
Although telomerase is an almost universal target for cancer therapy, there has been no effective telomerase targeted inhibitor that has progressed to late stage human clinical trials. Recently, we reported that a telomerase-mediated telomeredisrupting compound, 6-thio-2'-deoxyguanosine (6-thio-dG), was very effective at targeting telomerase positive cancer cells while sparing telomerase silent normal cells. 6-thio-dG, a nucleoside analogue of the already-approved drug 6-thioguanine, is incorporated into telomeres by telomerase, resulting in disruption of the telomereprotecting shelterin complex. This disruption leads to Telomere dysfunction-Induced Foci (TIFs) formation and rapid cell death for the vast majority of cancer cells. Since most chemotherapies eventually fail due to drug acquired resistance, novel drugs such as 6-thio-dG, as a single first line agent or in the maintenance setting, may represent an effective new treatment for cancer patients.
\end{abstract}

\section{INTRODUCTION}

At the end of linear chromosomes, repetitive sequence structures called telomeres are protected from being recognized as random DNA double strand breaks, which would otherwise activate DNA damage signaling responses. These sequences, (TTAGGG)n in mammals, are responsible for genomic stability by preventing recombination, end-end fusions/degradation and are also important in completing DNA replication during each cell cycle [1]. If telomeres become too short or "uncapped", chromosome ends cannot be properly protected by the shelterin proteins that bind to telomeric repeats [2]. This results in formation of breakage-fusionbridge cycles that lead to DNA double strand breaks and unstable multicentric chromosomes [3]. Under ideal culture conditions, growing normal cell populations eventually undergo senescence (growth arrest) due to progressive telomere shortening. A subset of cells can undergo cell death by bypassing senescence and entering crisis (where telomeres are even shorter compared to senescence). However, if rare cells escape crisis by loss of tumor suppressor gene functions, they acquire additional genomic changes. Importantly, these alterations in turn reactivate the ribonucleoprotein enzyme telomerase, which is present in $\sim 90 \%$ of primary human tumors but not in most somatic tissue cells, with the exception of transiently proliferating stem-like cells. Therefore, telomerase is a highly attractive, almost universal, target for cancer therapy $[4,5]$.

While there have been many different approaches to directly or indirectly target telomerase, only Imetelstat (GRN163L) has progressed to late stage human clinical trials. One concern with Imetelstat is the development of hematological toxicities requiring drug holidays that enable telomere re-elongation. An effective inhibitor would ideally permit long-term, robust ( $>99 \%$ ) telomerase inhibition or telomere dysfunction and rapid tumor shrinkage. Direct telomerase inhibitors in clinical trials do not show rapid tumor shrinkage not has robust telomerase 
inhibition been demonstrated. This is important since we have shown that only one percent of telomerase activity in cancer cells is sufficient to maintain the shortest telomeres and permit cells to continue to divide [6]. It is well established that telomerase preferentially elongates the shortest telomeres [7].

Hence, we [8] and others [9] have sought to develop new approaches to targeting telomeraseexpressing cancer cells. A base-modified nucleoside 6-thio-2'-deoxyguanosine (6-thio-dG) is an analogue of an already approved drug, 6-thioguanine. We reasoned that its 5'-triphosphate (formed in situ in cells) may be a telomerase-directed telomere uncapping compound. 6-thio-dG is rapidly converted to telomerase substrate 6-thio-2'-deoxyguanosine-5' -triphosphate (6-thio-dGTP) and consequently uses telomerase for its incorporation into telomeres. The guiding concept for initial proof-ofprinciple studies was that, since 6-thio-dG is converted rapidly into 6-thio-dGTP, it is potentially incorporated into both genomic DNA (by DNA polymerases) and telomeric DNA (by telomerase). We therefore predicted that 6-thio-dG would be both a more effective agent compared to 6-thioguanine and also induce cancer cell killing much more rapidly than a "classic" telomerase inhibitor. Once 6-thio-dG is incorporated into telomeres, the telomere sequence TTAGGG is modified at guanine bases, resulting in uncapping of telomeric DNA and likely loss of recognition and dissociation of the shelterin proteins from the de-novo formed modified telomeres. This leads to Telomere dysfunction Induced Foci (TIF) formation and rapid growth arrest or cell death of telomerase-positive cells. This relatively fast anti-cancer effect of 6-thio-dG has an important advantage compared to other direct telomerase inhibition approaches. Long and frequent treatment cycles of direct telomerase inhibitionbased therapies can cause side effects, as evidenced in some anti-telomerase clinical trials [10]. Importantly, the long lag period from initiation of treatment until cell death is dependent on initial cancer cell telomere length. Thus, patients with relatively long telomeres would require longer treatment periods and may be therapeutically disadvantaged by a direct telomerase inhibitor that is unlikely to stop the growth of the tumor cells before tumor burden is so extensive that there is no overall patient survival advantage. However, 6-thio-dG, a telomerase-mediated telomere uncapping agent, exerts a much more rapid effect, with a markedly reduced lag period. Importantly, this effect appears to be independent from initial cancer cell telomere lengths, as anticipated based on the compound's mechanism of action (Figure 1). In our studies, we have also demonstrated 6-thio$\mathrm{dG}$-induced telomere dysfunction in hTERT expressing cancer cells, as well as rapid and progressive telomere shortening in cancer cells that survive the initial treatment. Importantly, 6-thio-dG has minimal effects on normal human telomerase-silent cells. In addition, mice

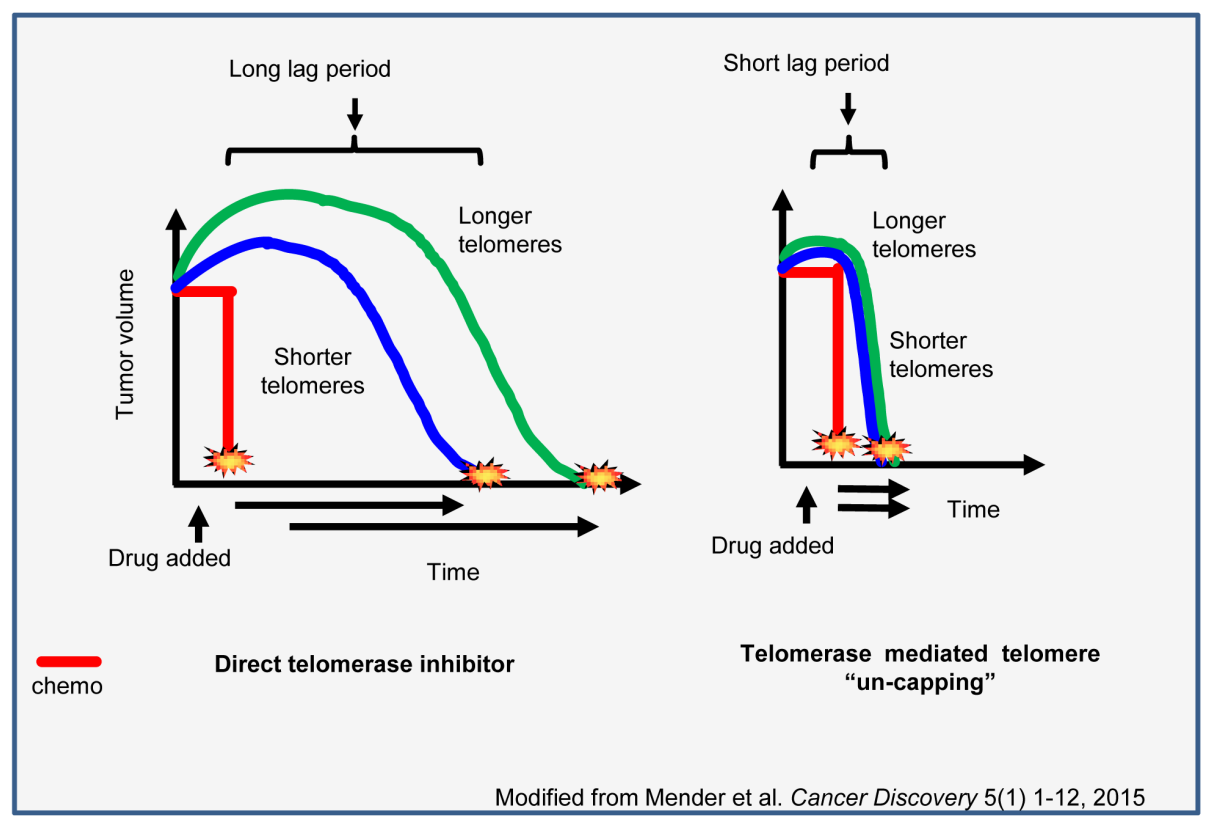

Figure 1: Comparison of two different approaches on telomerase targeted therapies. Since direct telomerase inhibitors cause telomere shortening by inhibiting telomerase activity, their effect will be depend on initial telomere length of cancer cells. While these inhibitors will have a long time period to shorten telomeres and be effective on cancer cells with long telomeres, this time period will be shorter for the cancer cells with shorter telomeres. One of the main concerns for the direct telomerase inhibitor approach is that the long treatment period may cause side effects on patients and they will need to stop therapy, which will lead to telomere re-elongation. However, since a telomerase-mediated telomere "un-capping" approach is independent from initial telomere length we expect to have a much more rapid effect on cancer cells leading to tumor shrinkage. 
that have been treated up to one month with therapeutic concentrations of 6-thio-dG do not lose weight and hematological, liver and kidney functions remain in the normal range. At the same time, in normal human fibroblasts with transfected with hTERT (e.g. ectopically introduced telomerase activity), 6-thio-dG induces TIFs and cell death, demonstrating a direct relationship between telomerase, 6-thio-dG and induction of TIFs. These results are independent from direct inhibition of telomerase activity in vitro, since 6-thio-dG does not inhibit telomerase activity in cancer cells, but instead uses telomerase's preferential incorporation of 6-thio-dG into telomeres to induce telomere uncapping. Moreover, while 6-thio-dG treatment causes cell death for the vast majority of cancer cells tested, normal fibroblasts and normal human colonic epithelial cells were largely unaffected. These results represent an attractive chemotherapeutic approach to primarily target telomerase expressing cancer cells, sparing normal cells. Indeed, in a small percent of cancer types that do not engage telomerase, these ALT (Alternative Lengthening of Telomeres) cells were also affected by 6-thio-dG, showing that general DNA damage of 6-thio-dG can lead to cell death, which, however, takes place at higher concentrations compared to effects on telomerase positive cells. Additionally, lung cancer cell based xenograft model studies showed dramatic tumor reduction, as well as telomere dysfunction in vivo induced by 6 -thio-dG treatment.

In summary, application of 6-thio-dG, with its proposed telomere-targeting mechanism of action, appears to be a promising anti-cancer treatment approach. As with any chemotherapy treatment, 6-thio-dG resistance mechanisms are expected to emerge. Understanding these resistance mechanisms may lead to more personalized based therapeutic regimens. In preliminary studies, we have tested a series of commonly-used multi-drug resistant cell lines and found 6-thio-dG to be active and effective in a significant fraction of these cell lines. Thus, 6-thio-dG may be effective in the maintenance setting after first line chemotherapy, with treatment yielding long-term durable responses for cancer patients.

\section{CONFLICT OF INTEREST}

The authors declare that they have a patent on 6-thio-dG which is licensed to Elizabeth Therapeutics, LLC.

\section{FUNDING}

I. Mender was supported by Scientific and Technological Research Council of Turkey (TUBITAK). These studies were supported in part by NCI SPORE P50CA70907, the Simmons Cancer Center Support Grant 5P30 CA142543 and support from the Southland Financial
Corporation Distinguished Chair in Geriatric Research (J.W.S. and W.E.W.). This work was performed in space constructed with support from National Institute of Health grant C06 RR30414. We thank Dr. Cynthia Lander for valuable discussions.

\section{REFERENCES}

1. Moyzis RK, Buckingham JM, Cram LS, Dani M, Deaven LL, Jones MD, Meyne J, Ratliff RL and Wu JR. A highly conserved repetitive DNA sequence, (TTAGGG)n, present at the telomeres of human chromosomes. Proceedings of the National Academy of Sciences of the United States of America. 1988; 85(18):6622-6626.

2. de Lange T. Shelterin: the protein complex that shapes and safeguards human telomeres. Genes \& development. 2005; 19(18):2100-2110.

3. Griffith JD, Comeau L, Rosenfield S, Stansel RM, Bianchi A, Moss $\mathrm{H}$ and de Lange T. Mammalian telomeres end in a large duplex loop. Cell. 1999; 97(4):503-514.

4. Kim NW, Piatyszek MA, Prowse KR, Harley CB, West MD, Ho PL, Coviello GM, Wright WE, Weinrich SL and Shay JW. Specific association of human telomerase activity with immortal cells and cancer. Science. 1994; 266(5193):20112015.

5. Shay JW and Bacchetti S. A survey of telomerase activity in human cancer. European journal of cancer. 1997; 33(5):787791.

6. Ouellette MM, Liao M, Herbert BS, Johnson M, Holt SE, Liss HS, Shay JW and Wright WE. Subsenescent telomere lengths in fibroblasts immortalized by limiting amounts of telomerase. The Journal of biological chemistry. 2000; 275(14):10072-10076.

7. Steinert S, Shay JW and Wright WE. Transient expression of human telomerase extends the life span of normal human fibroblasts. Biochemical and biophysical research communications. 2000; 273(3):1095-1098.

8. Mender I, Gryaznov S, Dikmen ZG, Wright WE and Shay JW. Induction of telomere dysfunction mediated by the telomerase substrate precursor 6-thio-2'-deoxyguanosine. Cancer discovery. 2015; 5(1):82-95.

9. Kim MM, Rivera MA, Botchkina IL, Shalaby R, Thor AD and Blackburn EH. A low threshold level of expression of mutant-template telomerase RNA inhibits human tumor cell proliferation. Proceedings of the National Academy of Sciences of the United States of America. 2001; 98(14):7982-7987.

10. Chiappori AA, Kolevska T, Spigel DR, Hager S, Rarick M, Gadgeel S, Blais N, Von Pawel J, Hart L, Reck M, Bassett E, Burington B and Schiller JH. A randomized phase II study of the telomerase inhibitor imetelstat as maintenance therapy for advanced non-small-cell lung cancer. Annals of oncology : official journal of the European Society for Medical Oncology / ESMO. 2015; 26(2):354-362. 